\title{
Psycho-social outcome in liver transplanted children: beware of emotional self-assessment!
}

\author{
Ana Maria Calinescu ${ }^{1,3}$, Valérie A McLin², Dominque Bellii, ${ }^{2,4}$ and Barbara E Wildhaber ${ }^{1,3^{*}}$
}

\begin{abstract}
Background: Psycho-social outcome in children after liver transplantation (LT) is known to be inferior to agerelated peers. Yet, when children and their parents are questioned by their nurse or physician about the child's psycho-social well-being, the answers usually are very positive. We hypothesized that patients and their parents after LT report their psycho-social well-being too enthusiastically when enquired by their personal care takers.

Methods: Inclusion criteria: LT at the Children's University Hospital of Geneva 1992-2007, age >3 years, <16 years, time after $L T>2$ years. Children and their parents were questioned by their well-known, familiar nurse at the annual follow up visit about their personal well-being. To allow for evaluation of answers, scores (good, medium, bad) were attributed to the different questions. 46 children were included in the study.

Results: Mean age at enquiry was 9.7 years (SD 4 years), mean time after LT was 7.5 years (SD 4.2 years). The different themes were reported as good for: parent-child relationship (83\%), relationship with peers (98\%), relation with siblings (39\%), sport activities (54\%), play activities (78\%), school performance (87\%), expression skills (67\%), and general behavior (89\%).

Conclusion: Most of our LT children and their parents consider, during a personal interview with a closely related, familiar nurse, that the child's psycho-social outcome is good. Yet, it is generally acknowledged that children after LT have negatively altered psycho-social outcomes. Thus, emotionally influenced reports about psycho-social outcome in children after LT must be looked at with care.
\end{abstract}

Keywords: Psycho-social outcome, Pediatric liver transplantation, Quality of life

\section{Introduction}

When evaluating the success of our liver transplantation (LT) program, we mainly used to take into account the patient's survival (90\%) and graft survival (82\%), our hospital being the only Swiss centre performing LT in children [1]. Yet, nowadays more and more attention is paid to the assessment of the health related quality of life (HRQOL) of our pediatric liver transplanted patients $[2,3]$. In the near future outcomes in LT might even be judged by the quality of life of the years restored by LT, a measure that might comprise both quality of life and survival rates [4].

\footnotetext{
* Correspondence: barbara.wildhaber@hcuge.ch

'Division of Pediatric Surgery, Department of Pediatrics, University Children's Hospital of Geneva, Geneva, Switzerland

${ }^{3}$ Service de Chirurgie Pédiatrique, Département de l'enfant et de l'adolescent, Rue Willy Donzé 6, 1211, Genève / Suisse, Switzerland

Full list of author information is available at the end of the article
}

The quantification of HRQOL in these patients remains a matter of debate. There is no "gold standard" that might measure the different concerns specific to this patient population [2,3]. Further, HRQOL needs to be assessed from several points of view: physical health, mental health, social functioning, role functioning and general health perception [5]. Investigators that previously examined this subject have clearly shown a lower HRQOL in liver transplanted children reported to normal healthy patients and equal to patients with chronic illness [6-9]. This is of most importance, since children with lower HRQOL will show impaired compliance and lower adherence to medical treatment, and thus may present with worse clinical outcome than those who are happy and psycho-socially stable [10-14].

The present study was conducted to challenge the psycho-social outcome of our Swiss pediatric liver transplantation population. We aimed to identify whether the

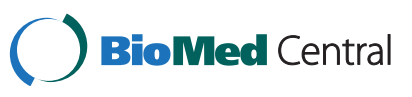


perception of the patient's well being, when inquired by the medical staff at the annual follow-up visit, is correct. Taking into account that, in the past, their answers usually had been very positive, we hypothesized that liver transplanted children and their parents report their psycho-social well being (too) enthusiastically when questioned by their personal, familiar medical caretakers.

\section{Methods}

\section{Study population}

Patients aged 3 to 16 years who underwent LT between 1992 and 2007 at the University Children's Hospital of Geneva, with a survival of more than two years after LT and receiving a follow-up care in University Children's Hospital of Geneva, were included in the study. A minimum of two years after LT was thought to be appropriate, since it has been shown that psycho-social outcomes significantly improve in the first six months after LT and remain rather stable thereafter [5]. Upon review of our local LT database we identified 46 children, all of them being included in the final study population. The study was approved by the Ethical Committee of the University Hospitals of Geneva.

\section{Study design}

The present qualitative study is a single centre cross sectional study. We used a semi-structured interview (Table 1), conducted by the transplant team nurse at the annual follow up visit. One of the or both parents together with the child were questioned by their wellknown, familiar nurse before the clinical assessment.

\begin{tabular}{l} 
Table $\mathbf{1}$ Questionnaire used in the semi-structured \\
interview \\
\hline A Social situation \\
How is the relationship with parents, siblings and peers? \\
How could you describe your playing activities? \\
Illness / liver transplantation \\
Medical condition? \\
Are you physically active? \\
Do you think that the liver transplantation limited your physical \\
activities? \\
Are you satisfied with your body? \\
Education \\
Have you finished primary school, high school? \\
Do you have school retardation? \\
Do you have career plans? \\
Psychological status \\
How would you describe your general behaviour? \\
Do you think that you are expressing yourself properly?
\end{tabular}

The order of asking the questions was random. The time for the interview was 30 to 40 minutes. A semistructured questionnaire was used in order to allow flexibility for the nurse, and to let new questions to be brought up during the interview, as a result of what the answers were, but still giving a framework of themes to be explored.

\section{Analysis}

Scores (good, medium and bad) were attributed to the different questions from our questionnaire to allow for evaluation of the answers. For analysis, the statistical programme SPSS 18 was used.

\section{Results}

Of the 46 children 27 (59\%) were transplanted for biliary atresia, $13(28 \%)$ for other cholestatic liver disease, 4 (9\%) for metabolic disease and 2 (4\%) for fulminant hepatitis. Mean age at inquiry was 9.7 years (standard deviation 4 years), mean time after LT was 7.5 years (standard deviation 4.2 years).

The parent child relationship was described as good in 38 cases (82.6\%), medium ("tense") in 7 (15.2\%) cases, and bad in 1 case $(2.2 \%)$. The relationship with peers was seen as good in $45(97.8 \%)$ children, as medium ("conflictual") in 1 case (2.2\%), and no child described a bad relationship with peers. The relationship with siblings was considered as good in 18 (39.1\%) children and as medium for 10 (39.1\%) children. Of note, 10 children and parents didn't want to or couldn't express their opinion on this topic. The physical stamina when performing sports was described as good in 25 (54.3\%) children, as medium in $16(34.8 \%)$ and bad for 5 patients (10.9\%). Play activities was thought to be good in 36 (78.3\%) patients, in 10 (21.7\%) as medium, no patient/ parent described a bad playing activity. Regarding educational data, the school activity was perceived as being good in $40(87 \%)$ patients and bad in $6(13 \%)$. Of note, this item was measured in years of school retardation: if no retardation was mentioned it was considered as good; at the presence of retardation it was classified as bad. The social expressional skills were considered as good in $31(67.4 \%)$ patients, as medium in $12(26.1 \%)$ and as bad in 3 patients $(6.5 \%)$. The patient's general behavior was seen as good in $41(89.1 \%)$ patients and as medium in 5 (10.9\%). No patient or parent described a bad behavior. Results are graphically summarized in Figure 1.

\section{Discussion}

It is generally accepted that outcomes after LT nowadays represent more than just survival rates: a child after LT does not only need physical follow-up, but also psychosocial evaluation [4]. In order to have a rigorous approach to the health status of LT patients physicians 


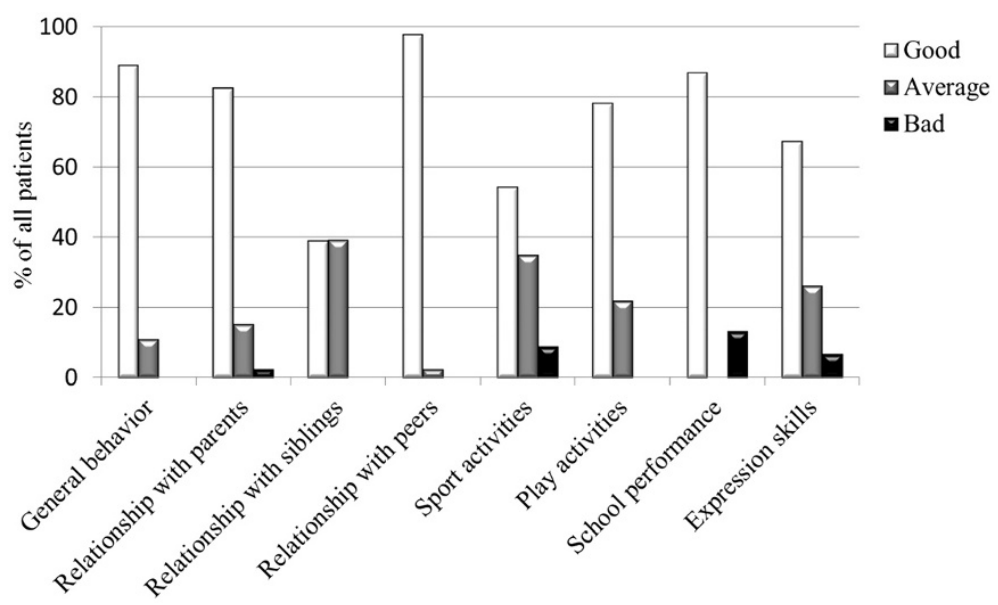

Figure 1 Most of our LT children and their parents consider, during a personal semi-structured interview with a closely related, familiar nurse, that the child's psycho-social outcome is good.

must examine not only physical but also the psychosocial outcomes [7]. Cross sectional studies show, for example, that psycho-social outcome in LT children is inferior to healthy peers and equal to children receiving cancer therapy [4]. Indeed, a LT child is not a cured, healthy child; it is a child for whom a fatal disease was substituted by a persistent, controlled, "ill-like" condition, which has associated morbidities that might be stable or evolve despite a well preserved allograft function $[2,3]$. Thus, children and their parents must face the burden of ongoing medical care and the anxiety about the child's future health [8]. In this particular context, the importance of evaluating the psycho-social outcomes is given: 1) by the fact that it is a way of hearing the child's own voice and perspective about his illness; 2 ) the measure of psycho-social outcomes might predict the future health and mortality of our patients [15]; and 3) an inferior psycho-social adjustment might be a cause as well as a result of non-adherence and non-compliance [14]. The non-adherence rate in pediatric LT recipients is about $30 \%$ and might contribute to $15 \%$ of graft loss [16]. Further, psycho-social evaluation may identify patients at risk for poor psycho-social long-term outcomes and allow us to offer anticipatory guidance and targeted interventions [4].

The measurement of HRQOL in LT children, and of their psycho-social outcome in particular, is controversial, as there is no "gold standard" instrument $[2,3]$. The tools we use must be age-specific and sensitive to developmental changes [16]. To date the instruments used are generic, allowing applicability across many types of disease, treatments and types of individuals. The use of disease specific tools would allow us to detect aspects relevant to LT children. For the younger age groups researchers only rely on parental assessment [9], but data must, whenever possible, be directly obtained from children, given the differences noticed between the child and parent reports [2,3]. The psycho-social assessment should be performed ideally by a mental health provider, familiar with the transplant scenario [11].

When our LT patients and their parents were inquired about their psycho-social outcomes by their well-known nurse at the annual follow up visit, their answers were very good in a striking majority. Knowing the latest literature in the field, our study must be considered as inaccurate to judge the real psycho-social status of our LT children. Our data suggest that emotionally influenced reports about psycho-social outcomes in children after LT must be interpreted with greatest care.

What are the reasons for the failure of this wellintended study? Children and parents may be excessively enthusiastic about their psycho-social well-being because of the challenges they have faced. They might underreport their difficulties for social desirability, e.g. wanting to give positive feedback to the team, and even for fear of poorer social integration. The familiar relationship between our nurses and children might also influence their response to please their personal medical caregiver.

There are some weaknesses of our study: We didn't carry out the same survey on a healthy, i.e. non-transplanted, control group. Still, we dare to assume that the answers from healthy children would have been comparable to the ones we obtained from our transplanted children and their parents, since almost all of those assume to do well - which we can suppose do healthy children, too. Further, we didn't analyze our children's HRQOL using a standardized questionnaire, as reported in the cited studies [6,7], which would have given us an "unbiased" control of the "real" HRQOL. Yet, even without these control groups this succinct clinical, observational 
study allows conclusions: The literature is clear and unequivocal, that is children after LT have a lower HRQOL [6-9]. This awareness is challenged after analysis of our questionnaire: our children and their parents believe that they do wonderfully well, even though the questions used in the interview were the same as asked in the reported literature; the only factor being different, was the fact that a personal, well-known nurse was present. The evidence based knowledge of the existing literature is an acceptable basis to allow us to conclude that our children and their parents were emotionally influenced during the interview. Nevertheless, further studies must be undertaken to demonstrate clear, valid evidence of our findings.

\section{Conclusion}

This succinct data suggests that the perception of the patient's well being, when inquired by the medical staff at the annual follow-up visit, seems incorrect. This study emphasizes the importance of routine standardized diagnostic procedures regarding cognitive and psycho-social development before and after transplantation in these children, as well as the importance of a professional mental health provider familiar with transplant issues being a necessary member of any transplant team in order to evaluate the real psycho-social outcomes of our liver transplanted children.

\section{Abbreviations}

LT: liver transplantation; HRQOL: health related quality of life.

\section{Competing interests}

The authors declare that they have no competing interests.

\section{Authors' contributions}

AC: Has made substantial contributions to the conception and design of the study, to the acquisition of data, and the analysis and interpretation of data; has been involved in drafting the manuscript. VMcL: Has been involved in revising the manuscript critically for important intellectual content. DB: Has been involved in revising the manuscript critically for important intellectual content. BEW: Has made substantial contributions to the conception and design of the study, and to the analysis and interpretation of data; has been involved in drafting the manuscript and revising it critically for important intellectual content; and has given final approval of the version to be published. All authors read and approved the final manuscript.

\section{Acknowledgements}

We would like to thank our transplant team nurse Lucianne AndreattaMerglen who performed each of the interviews at the annual follow up visit of our transplanted children.

\section{Author details}

'Division of Pediatric Surgery, Department of Pediatrics, University Children's Hospital of Geneva, Geneva, Switzerland. ${ }^{2}$ Unit of pediatric liver transplantation and hepatology, Department of Pediatrics, University Children's Hospital of Geneva, Geneva, Switzerland. ${ }^{3}$ Service de Chirurgie Pédiatrique, Département de l'enfant et de l'adolescent, Rue Willy Donzé 6, 1211, Genève / Suisse, Switzerland. ${ }^{4}$ Unité de Gastroentérologie et Hépatologie pédiatrique, Département de l'enfant et de l'adolescent, Rue Willy Donzé 6, 1211, Genève / Suisse, Switzerland.

\section{References}

1. Belli DC, Schaeppi M, Mentha G, Chardot C: Transplantations hépatiques chez l'enfant: série genevoise. Paediatrica 2006, 17(1):24-27.

2. Sundaram SS, Landgraf JM, Neighbors K, Cohn RA, Alonso EM: Adolescent health-related quality of life following liver and kidney transplantation. Am J Transplant 2007, 7(4):982-989.

3. Anthony SJ, Pollock Barziv S, Ng VL: Quality of life after pediatric solid organ transplantation. Pediatr Clin North Am 2010, 57(2):559-574.

4. Bucuvalas JC, Alonso E: Outcome after liver transplantation: more than just survival rates. Liver Transp/ 2005, 11(1):7-9.

5. Bucuvalas JC, Britto M, Krug S, Ryckman FC, Atherton H, Alonso MP, Balistrer WF, Kotagal U: Health-related quality of life in pediatric liver transplant recipients: A single-center study. Liver Transp/ 2003, 9(1):62-71.

6. Limbers CA, Neighbors K, Martz K, Bucuvalas JC, Webb T, Varni JW, Alonso EM: Health-related quality of life in pediatric liver transplant recipients compared with other chronic disease groups. Pediatr Transplant 2011, 15(3):245-253.

7. Alonso EM, Limbers CA, Neighbors K, Martz K, Bucuvalas JC, Webb T, Varni $J W$ : Cross-sectional analysis of health-related quality of life in pediatric liver transplant recipients. J Pediatr 2010, 156(2):270-276.

8. Alonso EM, Neighbors K, Barton FB, McDiarmid SV, Dunn SP, Mazariegos GV, Landgraf JM, Bucuvalas JC: Health-related quality of life and family function following pediatric liver transplantation. Liver Transp/ 2008, 14(4):460-468

9. Taylor R, Franck LS, Gibson F, Dhawan A: A critical review of the healthrelated quality of life of children and adolescents after liver transplantation. Liver Transp/ 2005, 11(1):51-60. discussion 57-59.

10. Fredericks EM, Magee JC, Opipari-Arrigan L, Shieck V, Well A, Lopez MJ: Adherence and health-related quality of life in adolescent liver transplant recipients. Pediatr Transplant 2008, 12(3):289-299.

11. Shemesh E: Assessment and management of psychosocial challenges in pediatric liver transplantation. Liver Transp/ 2008, 14(9):1229-1236.

12. Shemesh E, Annunziato RA, Shneider BL, Dugan CA, Warshaw J, Kerkar N, Emre S: Improving adherence to medications in pediatric liver transplant recipients. Pediatr Transplant 2008, 12(3):316-323.

13. Shemesh E, Shneider BL, Emre S: Adherence to medical recommendations in pediatric transplant recipients: time for action. Pediatr Transplant 2008, 12(3):281-283

14. Qvist $\mathrm{E}$, Jalanko H, Holmberg C: Psychosocial adaptation after solid organ transplantation in children. Pediatr Clin North Am 2003, 50(6):1505-1519.

15. Bucuvalas JC, Britto M: Health-related quality of life after liver transplantation: it's not all about the liver. J Pediatr Gastroenterol Nutr 2003, 37(2):106-108

16. Alonso EM: Quality of life for pediatric liver recipients. Liver Transp/2009, 15(Suppl 2):S57-62.

\section{doi:10.1186/1824-7288-38-37}

Cite this article as: Calinescu et al.: Psycho-social outcome in liver transplanted children: beware of emotional self-assessment!. Italian Journal of Pediatrics 2012 38:37.

\section{Submit your next manuscript to BioMed Central and take full advantage of:}

- Convenient online submission

- Thorough peer review

- No space constraints or color figure charges

- Immediate publication on acceptance

- Inclusion in PubMed, CAS, Scopus and Google Scholar

- Research which is freely available for redistribution 